ARTICLE

\title{
High-efficiency broadband achromatic metalens for near-IR biological imaging window
}

\author{
Yujie Wang ${ }^{1,5}$, Qinmiao Chen 1,5, Wenhong Yang 1,5, Ziheng Ji, ${ }^{1,5}$, Limin Jin (10 1, Xing Ma (1) 1, Qinghai Song (1) 1, \\ Alexandra Boltasseva (10 2 ${ }^{2}$, Jiecai Han ${ }^{3}$, Vladimir M. Shalaev (i) ${ }^{2} \&$ Shumin Xiao (1) 1,3,4凶
}

Over the past years, broadband achromatic metalenses have been intensively studied due to their great potential for applications in consumer and industry products. Even though significant progress has been made, the efficiency of technologically relevant silicon metalenses is limited by the intrinsic material loss above the bandgap. In turn, the recently proposed achromatic metalens utilizing transparent, high-index materials such as titanium dioxide has been restricted by the small thickness and showed relatively low focusing efficiency at longer wavelengths. Consequently, metalens-based optical imaging in the biological transparency window has so far been severely limited. Herein, we experimentally demonstrate a polarization-insensitive, broadband titanium dioxide achromatic metalens for applications in the near-infrared biological imaging. A large-scale fabrication technology has been developed to produce titanium dioxide nanopillars with record-high aspect ratios featuring pillar heights of $1.5 \mu \mathrm{m}$ and $\sim 90^{\circ}$ vertical sidewalls. The demonstrated metalens exhibits dramatically increased group delay range, and the spectral range of achromatism is substantially extended to the wavelength range of $650-1000 \mathrm{~nm}$ with an average efficiency of $77.1 \%-88.5 \%$ and a numerical aperture of $0.24-0.1$. This research paves a solid step towards practical applications of flat photonics.

\footnotetext{
${ }^{1}$ State Key Laboratory on Tunable laser Technology, Ministry of Industry and Information Technology Key Lab of Micro-Nano Optoelectronic Information System, Harbin Institute of Technology (Shenzhen), Shenzhen 518055, P. R. China. ${ }^{2}$ School of Electrical and Computer Engineering and Birck Nanotechnology Center, Purdue University, West Lafayette 47907 IN, USA. ${ }^{3}$ National Key Laboratory of Science and Technology on Advanced Composites in Special Environments, Harbin Institute of Technology, Harbin 150080, P. R. China. ${ }^{4}$ Collaborative Innovation Center of Extreme Optics, Shanxi University, Taiyuan 030006 Shanxi, P. R. China. ${ }^{5}$ These authors contributed equally: Yujie Wang, Qinmiao Chen, Wenhong Yang, Ziheng Ji.

凶email: shumin.xiao@hit.edu.cn
} 
$\mathrm{M}$ iniaturized untethered robots have been intensively studied due to their potential to bring disruptive advances to medical diagnostics and biological studies. Even though significant progress has been made in miniaturizing mobile untethered robots, their size for active imaging ranges from about 1 to $10 \mathrm{~mm}$. The main restriction for the microrobot size is in fact their on-board optical lenses. Consequently, the development of integrated optical lens has been a crucial step towards compact microrobots with on-board functionalities as well as other biomedical imaging techniques such as nano-optical endoscopy for high-resolution optical coherence tomography ${ }^{1-3}$. All-dielectric metalens that is a two-dimensional metamaterial consisting of a large number of dielectric nano-antennas ${ }^{4-6}$ offers intrinsic advantages for the realization of miniaturized integrated lens. Metasurface-based devices uniquely focus incident light to a diffraction-limited spot utilizing thin, flatform structures by precisely tailoring the wavefront ${ }^{7-12}$. High focusing efficiency and large numerical apertures (NA) have been demonstrated for a single wavelength soon after the invention of metalens ${ }^{13-16}$. Very recently, the chromatic aberration of metalenses have been successfully tackled too, making them attractive for active imaging ${ }^{17-23}$. The replacement of conventional bulk lenses with all-dielectric achromatic metalenses could address the longstanding challenge in on-board biomedical imaging.

In order to enable practical applications, several metasurface' characteristics must be carefully designed and optimized. To gain ultimate control over the phase of the propagating light, both the metalens' spatial and frequency-dependent phase profile must be controlled. The phase is given $b y^{5}$

$$
\varphi(r, \omega)=-\frac{\omega}{c}\left(\sqrt{r^{2}+F^{2}}-F\right)
$$

where $r, \omega$, and $F$ are the radial coordinates, angular frequency, and the focal length. To eliminate the chromatic aberration, both the phase $\varphi\left(r, \omega_{d}\right)$ and the group delay $\left.\frac{\partial \varphi}{\partial \omega}\right|_{\omega=\omega_{d}}$ around the central frequency $\omega_{d}$ must be properly controlled in the Taylor expansion of (1). The first term can be controlled by properly designing the resonant response, the Pancharatnam-Berry (PB) phase, or the propagation phase ${ }^{24-27}$. The latter can be manipulated with optimizing the waveguide mode by choosing suitable effective refractive index $n_{\text {eff }}$ and thickness $h^{26-28}$. Silicon that offers both high refractive index and mature nanofabrication technology, was shown to enable achromatic metalenses with efficiencies up to $50-60 \%$ in the spectral range from 1000 to $1800 \mathrm{~nm}$ (squares in Fig. 1) ${ }^{19}$. At shorter wavelengths above the band edge, the metasurfaces' performance was, however, strongly limited by the material absorption. Titanium dioxide $\left(\mathrm{TiO}_{2}\right)$ is another promising, technologically relevant and CMOS compatible material that can potentially overcome the absorption limitation of silicon ${ }^{17,18,29}$. However, current $\mathrm{TiO}_{2}$ metasurface fabrication techniques rely on the atomic layer deposition (ALD) method, which is relatively slow, and restricts the thickness and aspect ratio to the values below $600 \mathrm{~nm}$ and 15 , respectively ${ }^{15,17}$. Lalanne et al. ${ }^{30}$ pioneered an etching technique for $\mathrm{TiO}_{2}$ nanostructures with a height up to $990 \mathrm{~nm}$ and the first perspective for imaging ${ }^{31}$. The aspect ratio is still limited to $8.8-10$. As a result, the transmittance of nanopillar is usually sacrificed to fulfill the required group delay. For wavelengths above $630 \mathrm{~nm}$, achromatic metalens' focusing efficiency reduces rapidly to below $30 \%$ (dots in Fig. 1). While Ndao et al. ${ }^{32}$ reported an average efficiency of $\sim 70 \%$, they sacrificed the focusing ability of the demonstrated metalens and reduced the NA to 0.066. By combining recursive ray-tracing and simulated phase libraries, the hybrid achromatic metalens also shows its potential in achieving high focusing efficiency and broadband achromatism ${ }^{20}$. But the multi-photon process is not ready for shorter wavelength and the photoresist device faces the challenge from long-term durability. Therefore, metalens-based optical imaging in the biological transparency window $(650-1000 \mathrm{~nm})$ has so far been severely limited. Moreover, the efficiencies of the demonstrated achromatic metalenses are far below their monochromatic counterparts and cannot convincingly outperform the compound diffractive lens ${ }^{33-36}$. In this work, to address the above-mentioned challenges in metalens realization, we demonstrate high-efficiency, polarization-insensitive, and broadband achromatic metalens operating in the first biological imaging window.

\section{Results}

Working principle. In our work, we control the metalens characteristics by carefully designing the group delay, since the material's optical absorption is intrinsic and cannot be modified. The group delay range can be increased through simple geometry change, i.e., by making structures with larger aspect ratio/height. Here, we increase the pillar height to $h=1500 \mathrm{~nm}$, which is 2.5 times larger compared to the previous reports. Similar to ref. ${ }^{20}$, four types of nanopillars with circular-, ring-, square- and bipolar concentric ring-shaped cross-sections were employed as the metasurfaces building blocks (see Insets in Fig. 2a) to eliminate the polarization dependence. A parameter sweep of the nanopillar size and shape was done to build a library with the values of phase, group delay, and transmittance at the designed operational wavelength of $760 \mathrm{~nm}$ (see Supplementary Fig. 2). For an achromatic metalens with the diameter $D=30 \mu \mathrm{m}$ and $\mathrm{NA}=$ 0.24 , a particle swam optimization method was applied to optimize the nanostructures, minimizing the required group delay

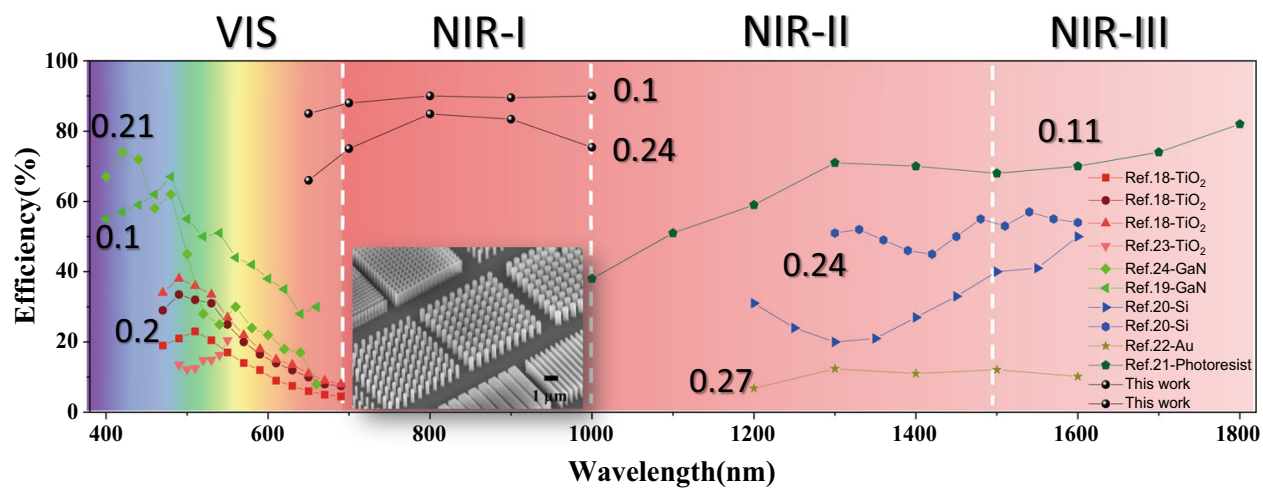

Fig. 1 Efficiency chart of the demonstrated broadband achromatic metalenses. The efficiencies of broadband achromatic metalenses with numerical

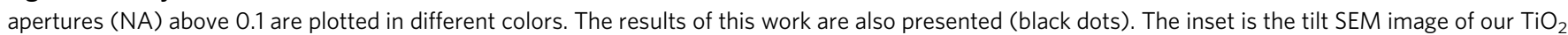
nanostructures with pillar height of $1500 \mathrm{~nm}$. 
a

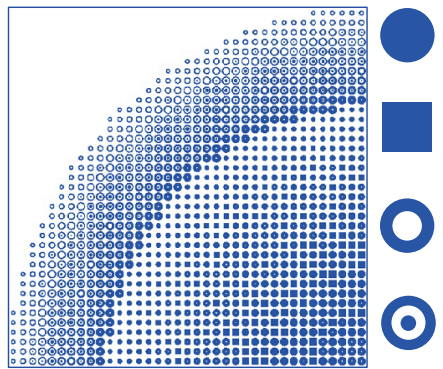

C

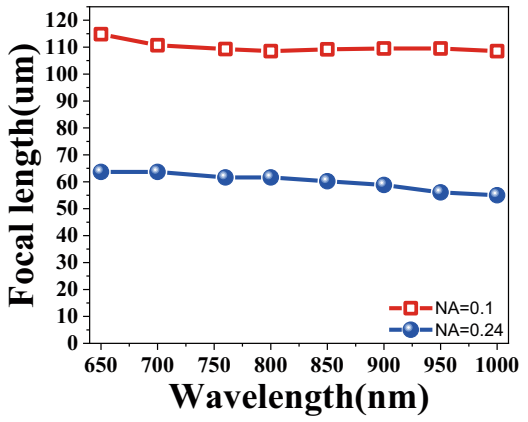

b
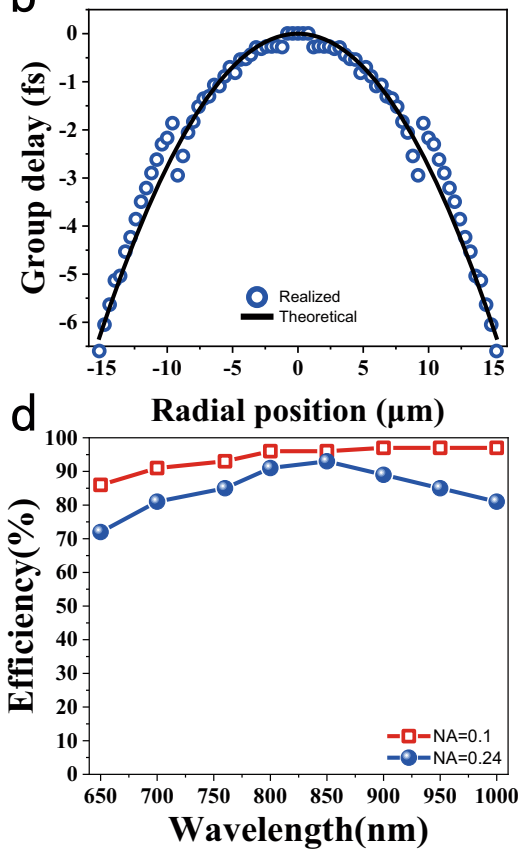

Fig. 2 Design of the near-IR achromatic metalens. a The layout of the quarter of the designed metalens. Four fundamental building blocks (unit cells) are enlarged in the insets. $\mathbf{b}$ The required group delay for broadband achromatism (solid line) and the values provided by the $\mathrm{TiO}_{2}$ nanostructures. $\mathbf{c}$ and $\mathbf{d}$ are the numerically calculated focal lengths and efficiencies of $\mathrm{TiO}_{2}$ metalenses with $\mathrm{NA}=0.24$ (dots) and $\mathrm{NA}=0.1$ (open squares) at different wavelengths. The diameters of two metalens are 30 and $25 \mu \mathrm{m}$, respectively.

range and maximizing the smallest feature size ${ }^{37}$. Figure 2 a shows the layout of a quarter of the developed metalens. It consists of four sets of $\mathrm{TiO}_{2}$ nanopillars with different cross-sections and a minimal feature size of $40 \mathrm{~nm}$. The required group delay (black curves) of the achromatic metalens was matched to the propagating group delay in nanopillars (dots in Fig. 2b). As a result, broadband achromatic performance was expected. Figure $2 \mathrm{c}$ shows the numerically calculated focal lengths. The incident light with the wavelength 650 to $1000 \mathrm{~nm}$, respectively, is focused by the $\mathrm{TiO}_{2}$ metalens to the designed position with a $<7 \%$ variation, covering the first biological imaging window. The corresponding focusing efficiency is plotted as dots in Fig. 2d. The minimal and maximal focusing efficiencies are $72 \%$ at $650 \mathrm{~nm}$ and $93 \%$ at $850 \mathrm{~nm}$, respectively. The averaged value in the entire operating spectrum is $83 \%$. Note that the average efficiency can be up to $93 \%$ for the small NA of 0.1 and diameter of $25 \mu \mathrm{m}$ (squares in Fig. 2d and details can be seen in Supplementary Fig. 3), surpassing all the current achromatic metalens demonstrations. In addition, as the nanopillars have at least fourfold rotational symmetry, the achromatic metalens is expected to be polarization insensitive, which is critical for biological applications ${ }^{38}$. Therefore, the developed $\mathrm{TiO}_{2}$ metalens with $h=1500 \mathrm{~nm}$ can potentially fill the so-called low-efficiency gap in available achromatic metalens designs.

Experiments. Experimental realization of $\mathrm{TiO}_{2}$ metalenses is a challenging task since the state of art pillar height is only $990 \mathrm{~nm}^{6,30}$. To overcome this challenge, we developed a topdown etching-based technology for fabricating $\mathrm{TiO}_{2}$ metalenses. First, 1500-nm-thick $\mathrm{TiO}_{2}$ membrane was deposited by the electron beam evaporation and coated with PMMA A2 resist, which was patterned with electron beam lithography. A lift-off process was applied to transfer the nanopatterns to $\mathrm{Cr}$ mask. Then, the membrane was etched with the reactive ion etching process (see details in Fig. 3a and "Methods"). After removing the
$\mathrm{Cr}$ mask, the $\mathrm{TiO}_{2}$ nanostructures were created. Figure 3b, c depicts the top-view scanning electron microscope (SEM) images of the developed $\mathrm{TiO}_{2}$ metalens. It consists of 4725 nanopillars with four types of different cross-sections. Note that the nanopillars locations, shapes and feature sizes closely follow the numerical design. The tilt-view SEM images of $\mathrm{TiO}_{2}$ metalenses in Fig. 3d, the inset in Fig. 1, and the Supplementary Fig. 5 show that the nanopillars have nearly perfect vertical sidewalls with the measured tilt angle of sidewalls of around $89^{\circ}-90^{\circ}$. Considering the smallest feature in the metalens, the achieved aspect ratio is around 37.5 or higher (Supplementary Fig. 5). The developed procedure for obtaining high-quality $\mathrm{TiO}_{2}$ nanostructures enabled the experimental realization of the metalens with the designed phase, group delay, and the corresponding highefficiency broadband achromatism.

The optical properties of the demonstrated $\mathrm{TiO}_{2}$ achromatic metalens were characterized using optical setup in Supplementary Fig. 6 . The focal lengths at different wavelengths were obtained by measuring the cross-sectional intensity profiles along the propagation direction (Fig. 3e). In the broad wavelength range from 650 to $1000 \mathrm{~nm}$, the incident light was focused to a bright spot $\sim 60 \mu \mathrm{m}$ away from the metalens with a small variation. The achieved values of both the focal length and the NA $=0.24$ match the numerical design very well.

To demonstrate the realization of a broadband achromatic metalens for the first biological imaging window, Fig. $3 \mathrm{f}$ shows the light intensity profiles in the $\mathrm{x}-\mathrm{y}$ plane at different wavelengths. The focal spot is a circular point at each wavelength. By fitting the intensity distributions along the diameter, all of the calculated Strehl ratios are larger than 0.81 and the full widths at half maximum (FWHM) deviate $<9 \%$ from the theoretical values, clearly demonstrating that the focal spots are diffraction limited. The 1951 United State Air Force (USAF) resolution test chart was used as a target to test the imaging capability of the developed metalens. For the incident light with the wavelength of $650 \mathrm{~nm}$, the metalens is able to resolve element- 4 of group 8 , giving a 
a

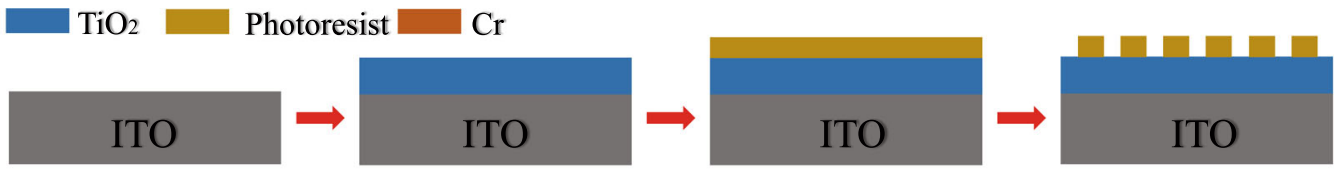

ITO glass Electron beam deposition Spin-coating photoresist EBL\&Development

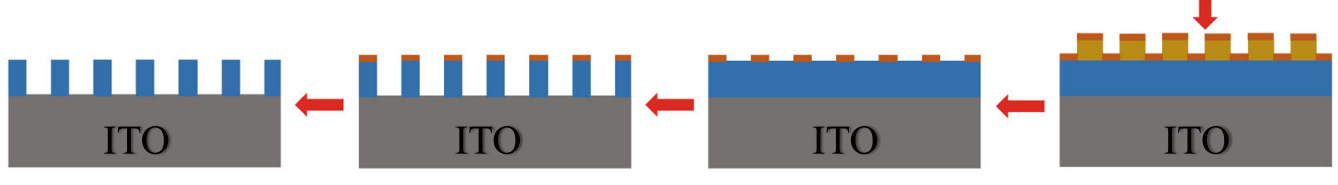

Cr removal

b

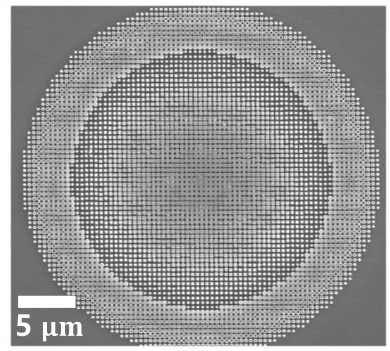

Etching

c

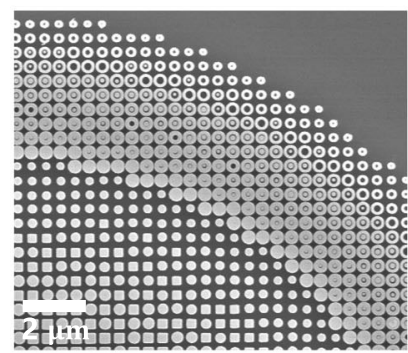

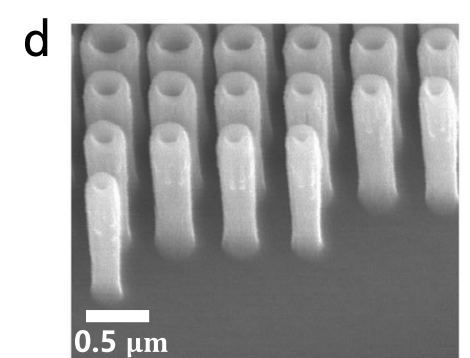

Deposition of Cr layer

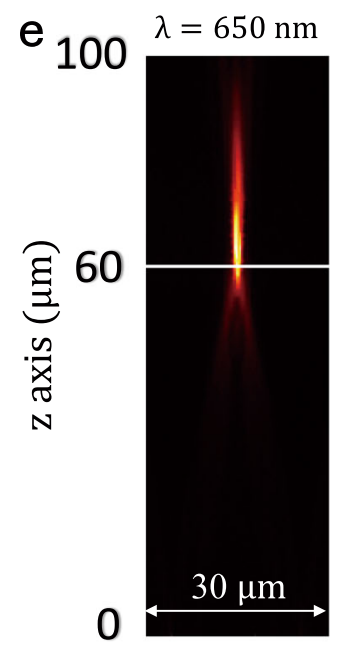

$\lambda=700 \mathrm{~nm}$

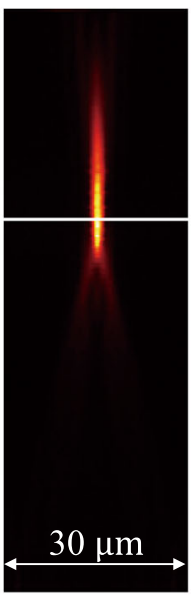

$f$
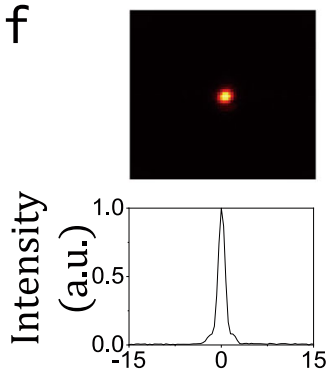

g
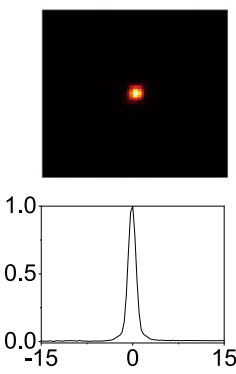
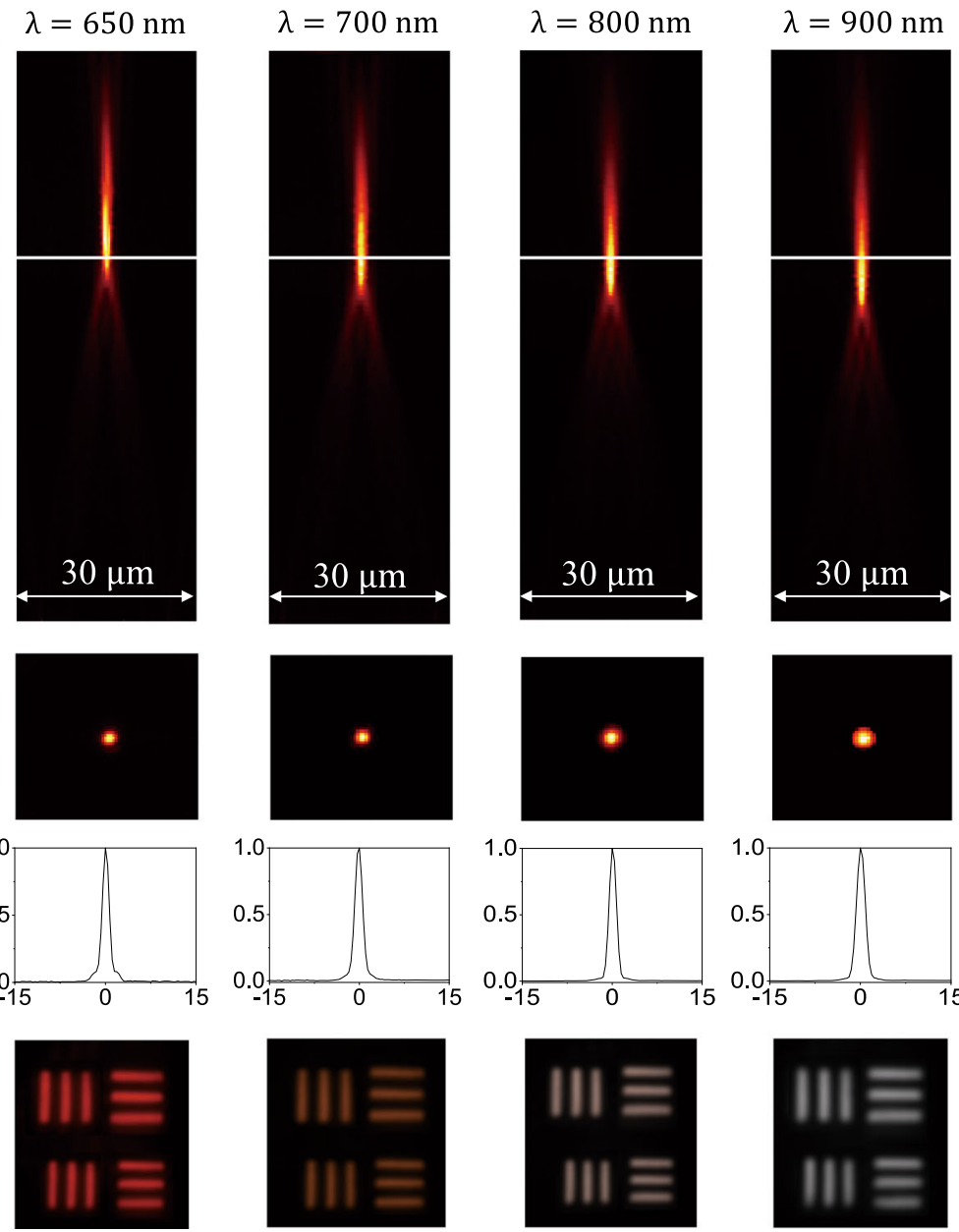

$\lambda=1000 \mathrm{~nm}$
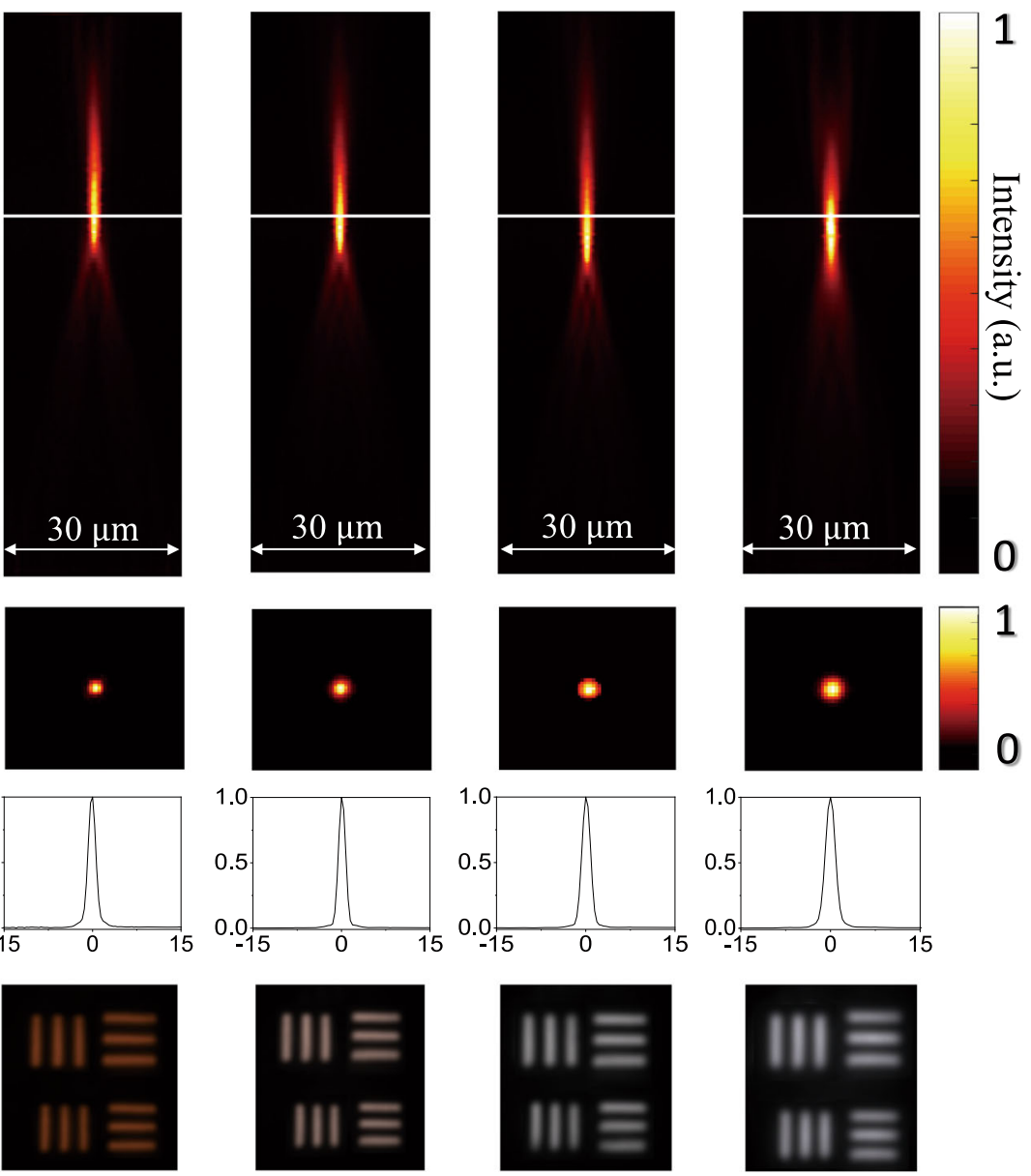

Fig. 3 The experimentally fabricated $\mathrm{TiO}_{\mathbf{2}}$ metalens with $\mathbf{N A}=\mathbf{0 . 2 4}$. a The schematic of the fabrication process. A highly directional etching process was employed to fabricate $\mathrm{TiO}_{2}$ nanostructures. $\mathbf{b}$ and $\mathbf{c}$ are the top-view SEM images of achromatic metalens with different resolutions. Four types of nanostructures can be clearly identified. $\mathbf{d}$ The corresponding tilt-view SEM image of the metalens. $\mathbf{e}$ and $\mathbf{f}$ are the intensity profiles of focal spots in $x-z$ plane and $x-y$ planes at different wavelengths. The bottom panels depict the intensity distributions along the diameter (solid lines) and the fitted curves (dashed lines). $\mathbf{g}$ The images of element-6, group-7 of the 1951 Unites States Air Force resolution target recorded by the achromatic metalens. 
a

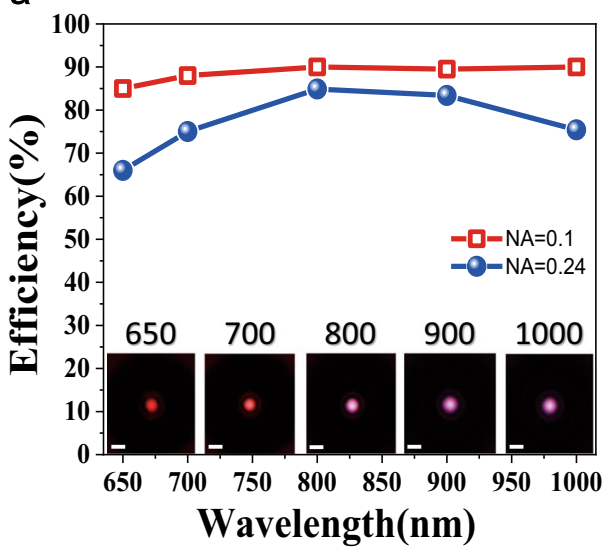

b

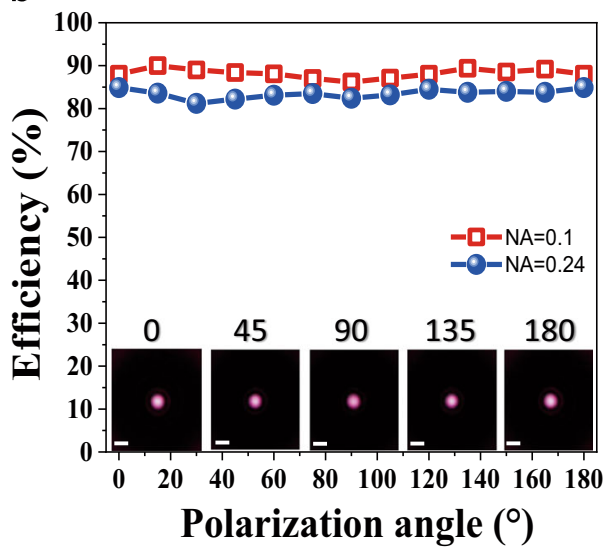

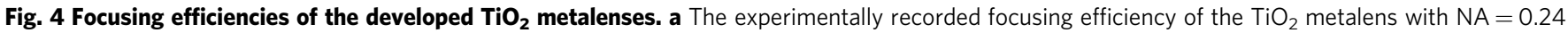
(dots) and NA $=0.1$ (open squares) as the function of the incident wavelength. Here, the incident light is un-polarized. $\mathbf{b}$ The dependence of the focusing efficiencies at $800 \mathrm{~nm}$ on the polarization. The insets in (a) and (b) are images of the focal spots of the metalens with $\mathrm{NA}=0.1$ at different wavelengths and different polarization, respectively.

resolution above $1.38 \mu \mathrm{m}$. While the resolution limit is dependent on the wavelength, the image of element- 6 of group 7 was clearly recorded at all wavelengths without tuning the metalens and the target (see Fig. 3g), confirming the broadband achromatism of our metalens very well.

Compared with the achromatism, the focusing efficiency is a more critical criterion for filling the near-IR gap. Similar to refs. ${ }^{2,8,9}$, we measured the efficiency by directly comparing the optical power of the focused light with the power of the incident light. The dots in Fig. 4a shows the experimentally recorded efficiencies of the $\mathrm{TiO}_{2}$ metalens with $\mathrm{NA}=0.24$. The lowest efficiency is $\sim 65 \%$ at $650 \mathrm{~nm}$, whereas the highest value is even $\sim 85 \%$ at $800 \mathrm{~nm}$. The averaged efficiency for the entire spectral range is $\sim 77.1 \%$, only a few percent lower than the numerical design. This is consistent with the high-quality fabrication of nanopillars since the efficiency reduces rapidly if the sidewall deviates from $90^{\circ}$ in the vertical direction.

Compared with the previous reports, the observed averaged efficiency is record-high. More interesting, we find that the averaged focusing efficiency of the developed $\mathrm{TiO}_{2}$ metalens can be furthered improved at smaller NAs. When the NA is 0.1 , the minimal and maximal focus efficiencies of the metalens at $650 \mathrm{~nm}$ and $700 \mathrm{~nm}$ are $85 \%$ and $90.2 \%$, respectively (see open squares in Fig. 4a and details in Supplementary Fig. 3). The averaged efficiency from 650 to $1000 \mathrm{~nm}$ is as high as $88.5 \%$. Since the Fresnel loss at the bottom and etched interfaces are not fully corrected, the averaged high-efficiency can reach above $90 \%$ that is superior to the commercial micro-lenses. According to the prediction in refs. ${ }^{4,5}$, such high-efficiency broadband achromatic $\mathrm{TiO}_{2}$ metalens can become a game changer in practical applications of flat photonics.

Polarization insensitivity is another important characteristic for applications in the near-IR window. Figure $4 \mathrm{~b}$ shows the focusing efficiencies of the demonstrated $\mathrm{TiO}_{2}$ metalenses at $800 \mathrm{~nm}$ at different polarization states. With the change of polarization, the efficiency of metalenses with $\mathrm{NA}=0.24$ and $\mathrm{NA}=0.1$ are almost flat at 85 and $89 \%$ for different linear polarization and circular polarization, consistent with the results of the non-polarized case in Fig. 4a. Similar polarizationinsensitive characteristics hold true for all other wavelengths (see Supplementary Fig. 8).

After we have demonstrated that the developed metalenses can successfully address the low-efficiency gap, we further explored the potential of $\mathrm{TiO}_{2}$ metalenses in upconversion imaging. In this experiment, the lanthanide-doped nanocrystals (NCs), which are widely used in bioimaging and labeling, were spread and aggregated on a glass substrate ${ }^{39}$. An additional layer of polystyrene (PS) spheres was deposited on top of the NC clusters. Under a conventional microscope, the NCs clusters were buried in PS spheres and cannot be identified (see Fig. 5a). Then, the continuous wave (CW) laser at $980 \mathrm{~nm}$ was focused by the $\mathrm{TiO}_{2}$ metalens onto the lanthanide-doped NCs clusters. The upconversion fluorescence centered at $655 \mathrm{~nm}$ with a linewidth of $20 \mathrm{~nm}$ was collected by the same metalens and recorded by a CCD camera (see Supplementary Fig. 9). By scanning the sample with a three-dimensional translation stage, the image can be reconstructed by combing the collected signals for each excitation point. The results are plotted in Fig. 5b. In contrast to conventional microscope image, the sharp edges of the NCs based microplate can be clearly seen in upconversion imaging. The resolution limit is around $1.46 \mu \mathrm{m}$, which is determined by the point spread function and the diffraction limit at the emission wavelength. For a direct comparison, the upconversion microscope image has been captured with a commercial achromatic objective lens with $\mathrm{NA}=0.26$ (Fig. $5 \mathrm{c}$ ). Both the metalens and the objective lens can capture the structural information of microplate with two-photon excitation with no obvious difference in resolution and intensity distribution (see Supplementary Figs. 10 and 11).

Potential application of the developed $\mathrm{TiO}_{2}$ achromatic metalens for biological imaging was also evaluated. For that, the HeLa cells containing lanthanide-doped NCs were prepared (Fig. 5d) and optically excited. Figure 5e depicts the fluorescent image of HeLa cells under two-photon excitation. Compared with the bright field microscope image in Fig. 5d, more detailed internal structures of the cell were captured. The image quality and the resolution were close to the ones recorded by the commercial objective lens with the similar NA (Fig. 5f). Thus, the demonstrated achromatic $\mathrm{TiO}_{2}$ metalens can perform on par with the commercial products for optical imaging. Considering its compactness, flatform, high-efficiency, polarization insensitivity, and diffraction-limit resolution, the proposed achromatic $\mathrm{TiO}_{2}$ metalenses could trigger a revolution in on-board biomedical diagnosis.

\section{Discussion}

In summary, we developed a top-down fabrication technique for the realization of superior quality, record-high aspect ratio $\mathrm{TiO}_{2}$ 

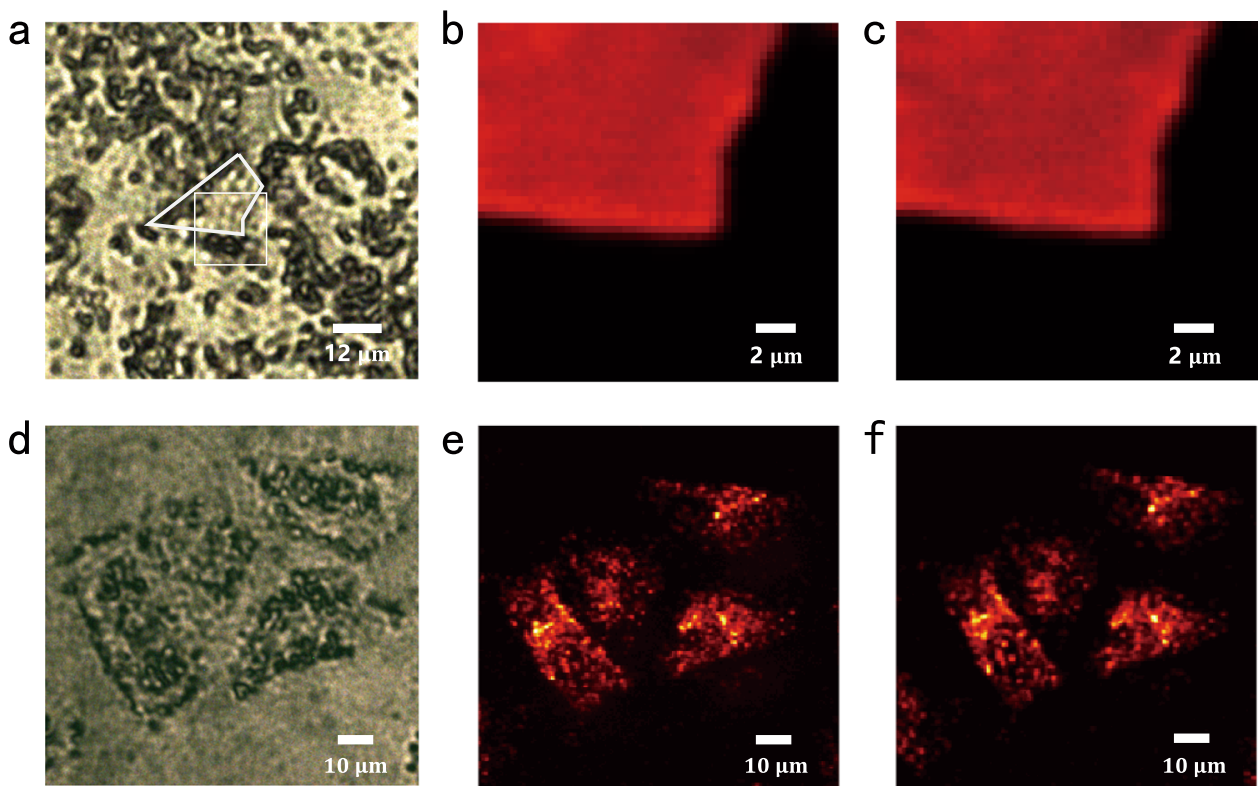

Fig. 5 Upconversion imaging with the metalens. a The microscope image of nanocrystals (NCs) on a microplate covered with polystyrene spheres under the white light illumination. The sample is marked with the dashed lines. $\mathbf{b}$ The upconversion fluorescent image recorded by the achromatic metalens with $N A=0.24$. c The upconversion fluorescent image recorded by a commercial achromatic objective lens with $N=0.26$ (MY10X-823, Mitutoyo). $\mathbf{d}$ The microscope image of HeLa cells with upconversion NCs. e and $\mathbf{f}$ and the upconversion fluorescent images recorded by the metalens and a commercial objective lens.

metasurfaces. With the increase in the metalens' thickness, the group delay range provided by the $\mathrm{TiO}_{2}$ nanopillars was dramatically enhanced. As a result, high-efficiency, broadband achromatic metalenses were designed and demonstrated in the near-IR biological window for an unpolarized incident light. The focusing efficiencies of the demonstrated achromatic metalenses were as high as $77.1 \%$ and $88.5 \%$ for the NA of 0.24 and 0.1 , respectively, while the maximal focusing efficiency was above $90 \%$. The proposed achromatic metalenses with record-high efficiencies and mass-manufacturable fabrication process could be extended to the visible spectrum as well (see Supplementary Fig. 12) and can potentially trigger a revolution in applications of flat optoelectronics ${ }^{17,40-43}$. Our work is also expected to accelerate the commercialization and biological applications of metasurfaces in portable devices as well as untethered microrobots.

\section{Methods}

Numerical simulation. The elements of phase-dispersion library were simulated by a finite element method-based commercial software COMSOL Multiphysics. Each element was simulated under linearly polarized illumination, and the propagation direction of light is along $z$-axis. The material properties of $\mathrm{TiO}_{2}$ and ITO-coated glass are all considered. Periodic boundary conditions were applied to the $\mathrm{x}$ - and $y$-directions and perfectly matched layer boundary conditions were applied to the $\mathrm{z}$-directions. For each simulation under sweep parameter with respect to the wavelength from 650 to $1000 \mathrm{~nm}$, then the phase spectrum was obtained. We linearly fit the phase spectrum of each element to obtain the group delay. To improve the achromatic performance, the achromatic metalens elements should have good linearity, then any element has an R-squared value $<0.995$ was dropped. In order to demonstrate the achromatic metalens focusing performance, the metalens was modeled in finite-domain time-difference simulations (Lumerical FDTD Solutions). Perfectly matched layer boundary conditions were used along the transverse and longitudinal directions and under plane wave illumination.

Deposition of $\mathrm{TiO}_{\mathbf{2}}$ film. The $\mathrm{TiO}_{2}$ film was deposited by electron beam evaporation. The ITO-coated glass substrate is cleaned and placed into the modified electron beam evaporator (Syskey, $30 \mathrm{kV}$ acceleration voltage). The vacuum pressure is pumped to $2 \times 10^{-7}$ Torr and the deposition rate is fixed at $0.6 \AA / s$. The $\mathrm{TiO}_{2}$ film with $1500 \mathrm{~nm}$ thickness is achieved after $6.94 \mathrm{~h}$ continuous deposition.

Fabrication of metalens. The metalens was fabricated using electron-beam lithography (EBL) on $1500 \mathrm{~nm}$ thick titanium dioxide film with quartz substrate to pattern the specific arrays. Firstly, PMMA A2 e-beam resist layer is deposited by spin-coating on titanium dioxide film. Then the sample is exposed through EBL and the patterns are revealed after the development process in MIBK/IPA. Subsequently, $30 \mathrm{~nm}$ thick $\mathrm{Cr}$ layer as hard mask deposited on sample used electrongun evaporator and lift-off process is done in solution of PG remover. Next, the patterns are transferred to titanium dioxide film by reactive ion etching (RIE) in Oxford 800 Plus. $\mathrm{SF}_{6}, \mathrm{CHF}_{3}$ and $\mathrm{O}_{2}$ are utilized in the etching process. The RF power is $100 \mathrm{~W}$ and the pressure is $9 \mathrm{mTorr}$. The ratio of $\mathrm{CHF}_{3} / \mathrm{SF}_{6}$ is tuned to 5.5 , effectively improving the anisotropy of etching. $\mathrm{O}_{2}$ is added to accelerate the etching speed in the vertical direction. The final sample can be obtained after removal of the $\mathrm{Cr}$ hard etching mask.

The optical characterization of metalens. The metalenses were characterized using a home-built microscope as shown in Supplementary Fig. 6. A collimated laser beam was converted into linearly polarized light after passing through the linear polarizer, Light exiting the polarizer is passing through the objective lens and incident on the metalens, then passes through another objective lens, and finally focuses on the CCD through a tube lens. The longitudinal intensity distribution of the metalens is acquired through a stack of $2 \mathrm{D}$ images by changing metalens position in predefined increments. The light intensity distribution of different wavelengths is obtained by inserting color filters.

For focusing efficiency measurement of the metalens, we measured the power in the focal spot (the power of transmission light passing through a circular area with radius of $3 *$ FWHM) and divided by the incident light power (the power of incident light passing through a circular area with radius same as metalens). Note that the efficiency can be further improved by fully correct the Fresnel loss the etched interface and the bottom interface.

\section{Data availability}

All the data supporting the findings of this study are available within the article, its Supplementary Information files, or accessed under the link https://pan.baidu.com/s/ 4lag6i6F.

Received: 17 March 2021; Accepted: 1 September 2021; Published online: 21 September 2021

\section{References}

1. Pahlevaninezhad, H. et al. Nat. Photon. 12, 540-547 (2018).

2. Fang, N., Lee, H., Sun, C. \& Zhang, X. Sub-diffraction-limited optical imaging with a silver superlens. Science 308, 534-537 (2005). 
3. Gissibl, T., Thiele, S., Herkommer, A. \& Giessen, H. Two-photon direct laser writing of ultracompact multi-lens objectives. Nat. Photon. 10, 554-560 (2017).

4. Khorasaninejad, M. \& Capasso, F. Metalenses: versatile multifunctional photonic components. Science 358, eaam8100 (2017).

5. Chen, W. T., Zhu, A. Y. \& Capasso, F. Flat optics with dispersion-engineered metasurface. Nat. Rev. Mater. 5, 604-620 (2020).

6. Lalanne, P. \& Chavel, P. Metalenses at visible wavelengths: past, present, perspectives. Laser Photon. Rev. 11, 1600295 (2017).

7. Kock, W. E. Metallic delay lenses. Bell Syst. Tech. 34, 321-339 (1948)

8. Stork, W., Streibl, N., Haidner, H. \& Kipfer, P. Artificial distributedindex media fabricated by zero-order gratings. Opt. Lett. 16, 1921-1923 (1991).

9. Ishii, S., Kildishev, A. V., Shalaev, V. M., Chen, K.-P. \& Drachev, V. P. Metal nanoslit lenses with polarization-selective design. Opt. Lett. 36, 451-453 (2011).

10. $\mathrm{Yu}, \mathrm{N}$. et al. Light propagation with phase discontinuities: generalized laws of reflection and refraction. Science 334, 333-337 (2011).

11. Ni, X., Emani, N. K., Kildishev, A. V., Boltasseva, A. \& Shalaev, V. M. Broadband light bending with plasmonic nanoantennas. Science 335, 427 (2012).

12. Lin, D., Fan, P., Hasman, E. \& Brongersma, M. L. Dielectric gradient metasurface optical elements. Science 345, 298-302 (2014).

13. Arbabi, A., Horie, Y., Ball, A. J., Bagheri, M. \& Faraon, A. Subwavelengththick lenses with high numerical apertures and large efficiency based on highcontrast transmit arrays. Nat. Commun. 6, 7069 (2015).

14. $\mathrm{Yu}, \mathrm{Y}$. F. et al. High transmission dielectric metasurface with $2 \pi$ phase control at visible wavelengths. Laser Photon. Rev. 9, 412-418 (2015).

15. Khorasaninejad, M. et al. Metalenses at visible wavelengths: diffraction-limited focusing and subwavelength resolution imaging. Science 352, 1190-1194 (2016).

16. Liang, $H$. et al. Ultrahigh numerical aperture metalens at visible wavelengths. Nano Lett. 18, 4460-4466 (2018).

17. Chen, W.-T. et al. A broadband achromatic metalens for focusing and imaging in the visible. Nat. Nanotechnol. 13, 220-226 (2018).

18. Wang, S. et al. A broadband achromatic metalens in the visible. Nat. Nanotechnol. 13, 227-232 (2018).

19. Shrestha, S., Overvig, A. C., Lu, M., Stein, A. \& Yu, N. Broadband achromatic dielectric metalenses. Light Sci. Appl. 7, 85 (2018).

20. Balli, F., Sultan, M., Lami, S. K. \& Hastings, J. T. A hybrid achromatic metalens. Nat. Commun. 11, 3892 (2020).

21. Wang, S. et al. Broadband achromatic optical metasurface devices. Nat. Commun. 8, 187 (2017).

22. Khorasaninejad, M. et al. Achromatic metalens over $60 \mathrm{~nm}$ bandwidth in the visible and metalens with reverse chromatic dispersion. Nano Lett. 17, 1819-1824 (2017).

23. Lin, R. J. et al. Achromatic metalens array for full-colour light-field imaging. Nat. Nanotechnol. 14, 227-231 (2019).

24. Bomzon, Z., Biener, G., Kleiner, V. \& Hasman, E. Space-variant Pancharatnam-Berry phase optical elements with computer-generated subwavelength gratings. Opt. Lett. 27, 1141-1143 (2002).

25. Kuznetsov, A. I., Miroshnichenko, A. E., Brongersma, M. L., Kivshar, Y. S. \& Luk'yanchuk, B. Optically resonant dielectric nanostructures. Science 354, aag2472 (2016)

26. Sauvan, C., Lalanne, P. \& Lee, M. S. L. Broadband blazing with artificial dielectrics. Opt. Lett. 29, 1593-1595 (2004)

27. Ribot, C. et al. Broadband and efficient diffraction. Adv. Opt. Mat. 1, 489-493 (2013).

28. Gigli, C. et al. Fundamental limitations of Huygens metasurfaces for optical beam shaping. Laser Photon. Rev. 15, 2000448 (2021).

29. Yoon, G., Kim, K., Huh, D., Lee, H. \& Rho, J. Single-step manufacturing of hierarchical dielectric metalens in the visible. Nat. Commun. 11, 2268 (2020).

30. Lalanne, P., Astilean, S., Chavel, P., Cambril, E. \& Launois, H. Design and fabrication of blazed binary diffractive elements with sampling periods smaller than the structural cutoff. J. Opt. Soc. Am. A 16, 1143-1156 (1999).

31. Lee, M. S. L. et al. Imaging with blazed-binary diffractive elements. J. Opt. A 4, s119-s124 (2002).

32. Ndao, A. et al. Octave bandwidth photonic fishnet-achromatic-metalens. Nat. Commun. 11, 3205 (2020).

33. Banerii, S. et al. Imaging with flat optics: metalenses or diffractive lenses? Optica 6, 805-810 (2019).

34. Engelberg, J. \& Levy, U. The advantages of metalenses over diffractive lenses. Nat. Commun. 11, 1991 (2020).
35. Mohammad, N., Meem, M., Shen, B., Wang, P. \& Menon, R. Broadband imaging with one planar diffractive lens. Sci. Rep. 8, 2799 (2018).

36. Wang, P., Mohammad, N. \& Menon, R. Chromatic-aberration-corrected diffractive lenses for ultra-broadband focusing. Sci. Rep. 6, 21545 (2016).

37. Chen, W. T., Zhu, A. Y., Sisler, J., Bharwani, Z. \& Capasso, F. A broadband achromatic polarization-insensitive metalens consisting of anisotropic nanostructures. Nat. Commun. 10, 355 (2019).

38. Arbabi, E. et al. Two-photon microscopy with a double wavelength metasurface objective lens. Nano Lett. 18, 4943-4948 (2018).

39. Zhu, H. et al. Amplified spontaneous emission and lasing from Lanthanidedoped uo-conversion nanocrystals. ACS Nano 7, 11420-11426 (2013).

40. Park, J.-S. et al. All-glass, large metalens at visible wavelength using deepultraviolet projection lithography. Nano Lett. 19, 8673-8682 (2020).

41. Kwon, H., Arbabi, E., Kamali, S. M., Faraji-Dana, M. \& Faraon, A. Single-shot quantitative phase gradient microscopy using a system of multifunctional metasurfaces. Nat. Photon. 14, 109 (2020).

42. Schlickriede, C. et al. Nonlinear imaging with all-dielectric metasurfaces. Nano Lett. 20, 4370-4376 (2020).

43. Rogers, E. T. F. et al. Far-field unlabeled super-resolution imaging with superoscillatory illumination. APL Photon. 5, 066107 (2020).

\section{Acknowledgements}

This research was supported by the National Key Research and Development Project (Grant No. 2018YFB2200403), National Natural Science Foundation of China (Grant Nos. 12025402, 11974092, and 11934012), Shenzhen Fundamental research projects (Grant No. JCYJ20180507184613841, JCYJ20180507183532343, JCYJ20200109112805990, and JCYJ20200109113003946), Shenzhen engineering laboratory on organic-inorganic perovskite devices, and the Fundamental Research Funds for the Central Universities(Grant No. HIT. BRET. 2021009). Purdue authors acknowledge support from the Air Force Office of Scientific Research (AFOSR) grant FA9550-18-1-0002 and FA9550-20-1-0124.

\section{Author contributions}

S.X. designed and supervised the experiment. Y.W. and W.Y. fabricated the samples Q.C. designed the achromatic metalens. Z.J. and Y.W. performed the optical characterization. L.J. and X.M. provided the upconversion nanoparticles and HeLa cells. Q.S., A.B., J.H., V.S. and S.X. discussed the results and prepared the manuscript.

\section{Competing interests}

The authors declare no competing interests.

\section{Additional information}

Supplementary information The online version contains supplementary material available at https://doi.org/10.1038/s41467-021-25797-9.

Correspondence and requests for materials should be addressed to Shumin Xiao.

Peer review information Nature Communications thanks the anonymous reviewer(s) for their contribution to the peer review of this work. Peer reviewer reports are available.

Reprints and permission information is available at http://www.nature.com/reprints

Publisher's note Springer Nature remains neutral with regard to jurisdictional claims in published maps and institutional affiliations.

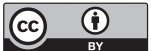

Open Access This article is licensed under a Creative Commons Attribution 4.0 International License, which permits use, sharing, adaptation, distribution and reproduction in any medium or format, as long as you give appropriate credit to the original author(s) and the source, provide a link to the Creative Commons license, and indicate if changes were made. The images or other third party material in this article are included in the article's Creative Commons license, unless indicated otherwise in a credit line to the material. If material is not included in the article's Creative Commons license and your intended use is not permitted by statutory regulation or exceeds the permitted use, you will need to obtain permission directly from the copyright holder. To view a copy of this license, visit http://creativecommons.org/ licenses/by/4.0\%

(c) The Author(s) 2021 